Cahiers
de la Recherche
sur les Droits

Cahiers de la recherche sur les droits

fondamentaux

$12 \mid 2014$

Droit et psychiatrie

\title{
Conclusions : la norme et l'anormal
}

Jean-Yves Carlier

\section{OpenEdition}

Journals

Édition électronique

URL : http://journals.openedition.org/crdf/1952

DOI : $10.4000 /$ crdf.1952

ISSN : 2264-1246

Éditeur

Presses universitaires de Caen

Édition imprimée

Date de publication : 1 décembre 2014

Pagination : 107-109

ISBN : 978-2-84133-507-7

ISSN : 1634-8842

Référence électronique

Jean-Yves Carlier, «Conclusions : la norme et l'anormal », Cahiers de la recherche sur les droits fondamentaux [En ligne], 12 | 2014, mis en ligne le 01 octobre 2015, consulté le 12 février 2020. URL : http://journals.openedition.org/crdf/1952; DOI : 10.4000/crdf.1952 


\title{
Conclusions: la norme et l'anormal
}

\author{
Jean-Yves CARLIER \\ Professeur aux universités de Louvain et de Liège (Belgique)
}

\section{Liberté \\ II. Égalité \\ III. Fraternité}

Bien sûr, il n'est plus de bon ton de parler d' «anormal». Il n'empêche, l'opposition des mots exprime bien le caractère improbable du couple «Droit et psychiatrie» qui forme le titre du colloque organisé le 15 novembre 2013 à l'université de Caen Basse-Normandie, sous la responsabilité scientifique conjointe de Marie-Joëlle Redor-Fichot (Centre de recherche sur les droits fondamentaux et les évolutions du droit) et Mathias Couturier (Centre de recherche en droit privé). C'est que le droit se veut norme alors que la psychiatrie, selon le sens commun, s'occuperait de l'anormal. Le droit a longtemps sacrifié à cette logique binaire. L'aliéné pouvait être considéré comme appartenant à autrui, étranger à lui-même, à l'image du non-national, étranger à la société étatique organisée. Si le colloque s'est attaché, par des contributions riches et précises, à analyser la manière dont le droit appréhende, aujourd'hui en France, la psychiatrie, il pourrait être intéressant de s'interroger également sur ce que, à rebours, la psychiatrie aurait à dire au droit. Et sans doute serait-ce, pour l'essentiel, de s'interroger sur cette notion d'extranéité - de l'étranger à l'étrange - et sur la logique binaire du droit qui tend à opposer le légal et l'illégal, le licite et l'illicite, le normal et l'anormal, le national et l'étranger, le public et le privé... Or, la complexité du réel est plus riche que ce classement formel, elle se nourrit aussi de zones grises et de marges. Si le droit doit demeurer ordonnancement et régulation de la vie en société, il doit affiner ses techniques de classement et adapter ses commandements et les mécanismes de leur mise en œuvre qui, plus qu'avant, tiennent compte d'une société complexe construite sur deux pôles en apparence opposés, la recherche d'une unité égalitaire (universalisme) et le respect de la diversité (particularisme) ${ }^{1}$. Dans différents domaines du droit, des évolutions considérables ont pris acte de cette complexité. Ainsi, pour ce qui concerne le sujet de ce colloque, la seule mesure d'interdiction du majeur frappé «d'imbécilité, de démence ou de fureur» prévue à l'article 489 du Code Napoléon a laissé la place à différents « régimes de protection» des «intérêts de la personne» «lorsque ses facultés mentales sont altérées par une maladie, une infirmité ou un affaiblissement dû à l'âge», selon l'article 490 du Code civil tel que libellé par la loi no 68-5 du 3 janvier 1968. Depuis l'entrée en vigueur le $1^{\text {er }}$ janvier 2009 de la loi $n^{\circ}$ 2007-308 du 5 mars 2007, l'article 415 du Code civil stipule que:

Les personnes majeures reçoivent la protection de leur personne et de leurs biens que leur état ou leur situation rend nécessaire $[\ldots]$.

Cette protection est instaurée et assurée dans le respect des libertés individuelles, des droits fondamentaux et de la dignité de la personne.

1. Voir M. Delmas-Marty, Le relatifet l'universel, Paris, Seuil, 2004; D. Lochak, Le droit et les paradoxes de l'universalité, Paris, PUF, 2010; F. Rigaux, Les situations juridiques individuelles dans un système de relativité générale. Cours général de droit international privé, in Recueil des cours de l'Académie de droit international de La Haye, t. 213, 1989, p. 9-407. 
Elle a pour finalité l'intérêt de la personne protégée. Elle favorise, dans la mesure du possible, l'autonomie de celle-ci.

Elle est un devoir des familles et de la collectivité publique.

L'évolution de l'imbécile au sujet de droit, de l'exclusion vers l'inclusion adaptée est évidente.

D'une certaine manière, les interventions au colloque «Droit et psychiatrie» ont montré en filigrane que l'évolution de l'appréhension du sujet de la psychiatrie comme sujet de droit correspondait à la trilogie républicaine sur laquelle peuvent encore se construire les démocraties contemporaines: liberté, égalité, fraternité.

\section{Liberté}

Sous le titre «Des lois sous influences", Jean-Manuel Larralde montre, jurisprudence à l'appui, à quel point la Cour européenne des droits de l'homme et le Conseil constitutionnel ont joué un «rôle [...] central dans [le] mouvement récent de rénovation du droit des hospitalisations psychiatriques ». La jurisprudence de Strasbourg, rappelant le principe de liberté inscrit à l'article 5 de la Convention européenne des droits de l'homme, impose à toute forme de privation de liberté, qui doit demeurer l'exception, de poursuivre un but légitime par des moyens proportionnés. C'est dire la "place essentielle du juge» dans le contrôle de ces conditions. Même en présence d'avis d'experts médicaux, la Cour européenne des droits de l'homme insiste sur la nécessité pour les juridictions nationales d'examiner en détail les motifs et la proportionnalité de la mesure privative de liberté ${ }^{2}$. Tel est le rôle en France du juge des libertés et de la détention (JLD). Depuis la réforme de 2011, il prend le pas sur le juge administratif. Ce dernier conserve toutefois une compétence dont le champ est devenu incertain. Marie-Joëlle Redor-Fichot note que «l'unification du contentieux opérée par la loi de 2011 est loin d'être complète, laissant ouverte la question de savoir ce qui relève encore de la compétence du juge administratif ». L'affaiblissement qui en résulte de la cohérence tant recherchée inviterait même à réinterroger ce système de répartition des compétences entre les deux ordres de juridiction au travers de l'article 66 de la Constitution. D'autre part, le JLD lui-même, dans sa pratique ou en sa qualité de gardien du bon fonctionnement du service du contentieux, comme Thierry Hanouët, a de légitimes motifs d'interrogation. L'intitulé même de la fonction de juge des libertés et de la détention correspond-il à l'objectif de soins, "surtout auprès d'un public fragile»? Plus fondamentalement, l'exercice de la même fonction à l'égard d'autres catégories de personnes - les délinquants et les étrangers - induit-il nécessairement des rapprochements positifs dans les raisonnements et décisions? L'objectif de garantir la liberté sans stigmatiser est-il atteint? Reconnaître l'égalité en est une condition.

\section{II. Égalité}

C'est en considérant le sujet de soins psychiatriques comme sujet de droit que son égalité est affirmée avec «toute personne», bénéficiaire des droits fondamentaux.

À cet égard, la contractualisation des rapports soignant-soigné et le rôle du consentement se sont développés. Mais plusieurs intervenants se sont interrogés: qui consent, à quoi et comment? Pour que le consentement soit libre et éclairé, la triangulation de la relation, dépassant le binôme soignant-soigné, paraît importante. Le tiers intervenant est alors souvent issu du monde judiciaire: juge, procureur, avocat. Mais il peut aussi faire partie des membres de la famille, expressément citée à l'article 415 du Code civil. Denis Malo, pour l'Union nationale des amis et familles de malades psychiques (UNAFAM), en rappelle l'importance. Il évoque, notamment, la possibilité d'« obtenir à l'audience une prise en compte systématique du point de vue de la famille quand elle le souhaite et sous la forme la plus opportune». L'égalité impose de construire chaque fois un dialogue institutionnalisé entre les différents acteurs dans le respect de la dignité humaine de la première personne concernée.

L'égalité de droits conduit aussi à l'égalité de responsabilités et d'obligations car «principe d'égalité oblige, les malades mentaux répondent surtout - comme toute personne qui jouit de sa pleine lucidité et de sa pleine capacité juridique - de leur faute personnelle», rappelle Gilles Raoul-Cormeil.

On perçoit que ce principe d'égalité, tant dans sa branche positive que négative, doit être lu à la lumière des adaptations aristotéliciennes. Il s'agit non seulement de traiter de façon identique ce qui est identique, mais aussi de façon différente ce qui est différent. En termes modernes, au sein de l'égalité entre toutes personnes, il y a lieu de tenir compte de la vulnérabilité de certaines catégories de personnes et d'adapter le droit en conséquence. C'est le rôle protectionnel à attribuer à la fraternité.

\section{Fraternité}

Le terme est désuet, on y préfère la solidarité. Mais celle-ci souffre autant que celle-là des œillères de l'individualisme. La loi doit alors remédier à la générosité vacillante et transformer le don en devoir de protection.

Sophie Théron, analysant le contenu de la loi du 5 juillet 2011, montre que la protection des droits des malades est considérablement améliorée. Il n'empêche, précise-t-elle avec lucidité, cette protection demeure «équivoque». C'est que, de tout temps, cette protection poursuit un objectif double et ambigu: «la nécessité de protéger non seulement la personne concernée mais aussi et surtout les tiers». Dit autrement, la société peut avoir tendance à se soucier davantage de sa propre protection que de la protection de la personne concernée. Gilles 
Raoul-Cormeil offre une analyse détaillée et documentée de la protection des biens et de la personne du malade mental. Cette protection doit être adaptée aux réalités et tenir compte du «triple principe: nécessité, subsidiarité, proportionnalité». Par exemple, plutôt que la mise sous tutelle ou curatelle,

Si le juge peut faire autrement, il devra privilégier les mesures alternatives que sont notamment la procuration, le mandat de protection future et la représentation judiciaire, si elles permettent de pourvoir aux intérêts de la personne vulnérable.

Mathias Couturier montre à quel point cette même notion de vulnérabilité apparaît en droit pénal comme cause d'aggravation, voire de constitution, de l'infraction à l'encontre d'un malade mental. Ce qui n'exclut pas, ambiguïté toujours, que le même droit pénal serve à «la protection de la société contre les risques générés par la santé mentale». C'est aussi, rappelle Philippe Salvage, que «la responsabilité pénale du malade mental» répond à des «principes de base» qui ne permettent pas de prononcer aisément une abolition ou une altération du discernement.

La notion de vulnérabilité prend une place de plus en plus importante dans le droit en général et dans les jurisprudences relatives aux droits de l'homme en particulier ${ }^{3}$. Cette consécration de l' " homo vulnerabilis ${ }^{4}$ n'est pas sans danger. Elle peut constituer une manière camouflée de cette charité bien ordonnée qui, commençant par soimême, exclut plus qu'elle n'inclut dans la société. Gilles Raoul-Cormeil rappelle ici les mots du doyen Carbonnier:

[...] légiférer sur les fous pour les fous, n'est-ce pas le pire? Très vite l'impression doit vous venir qu'on ne peut être pour eux, sans au fond, d'une certaine manière, agir contre eux. Car toute protection des aliénés, en un sens, les aliène, par cela seul qu'elle les suppose étrangers à l'univers raisonnable. Si bien que, procédant des meilleures intentions, une législation protectrice ne fera qu'ajouter de son métal à cet appareil répressif par lequel les sociétés modernes, avec des hypocrisies variables, rejettent de leur sein ceux qui ont le malheur d'avoir des mécanismes mentaux dissidents 5 .

Mais la vulnérabilité peut aussi devenir un concept structurel du droit, une manière de prolongement des idées de celui qui fut à l'origine des évolutions majeures du Code civil français en 1968, évoluant vers un «flexible droit ${ }^{6}$ et peut-être, aujourd'hui, vers un « sensible droit» selon la formule de Loïc Azoulai ${ }^{7}$. Cette conception structurelle de la vulnérabilité permet de la différencier de la simple protection de la fragilité en ce qu'elle comporte «une dimension relationnelle ${ }^{8}$. Elle structure la fraternité en solidarité.

La protection de la vulnérabilité est toutefois guettée par un autre danger ici rappelé par Mathias Couturier. C'est celui d'une nouvelle "normalité» contrôlée. D'une organisation généralisée du bonheur pour tous faisant de la santé mentale une manière de «nouvelle grammaire de l'organisation sociale» et de «reconfiguration du droit». Le «paradigme de la santé mentale» gestionnaire risquerait alors de conduire, à partir de «la négation de l'humanité de la folie [...] à une tendance persistante à la déshumanisation de l'existence ${ }^{9}$. Ce serait un exemple de ce "passage subreptice de l'être au devoir être», de la «tentation du scientisme qui a souvent entraîné les juristes à des affirmations non vérifiées ${ }^{10}$. Sans exagérer ce risque, il convient d'y être attentif.

On le disait, la psychiatrie a autant à dire sur le droit que l'inverse.

3. Voir La vulnérabilité saisie par les juges en Europe (actes du colloque organisé à l'École de droit de la Sorbonne le 7 décembre 2012), L. BurgorgueLarsen (dir.), Paris, Pedone (Cahiers européens; 7), 2014; Vulnerability: Reflections on a New Ethical Foundation for Law and Politics, M. A. Fineman, A. Grear (dir.), Farnham, Ashgate, 2013.

4. P. Martens, «La nouvelle controverse de Valladolid», Revue trimestrielle des droits de l'homme, 2014, p. 307.

5. J. Carbonnier, Essais sur les lois, $2^{\mathrm{e}}$ éd., Paris, Répertoire du notariat Defrénois, 1995, p. 63-78, spéc. p. 71; cité supra par G. Raoul-Cormeil.

6. J. Carbonnier, Flexible droit: textes pour une sociologie du droit sans rigueur, Paris, LGDJ, 1969.

7. L. Azoulai, «Sensible droit», in La vulnérabilité saisie par les juges en Europe.

8. P. Martens, «La nouvelle controverse de Valladolid», p. 327.

9. P. Coupechoux, Un homme comme vous. Essai sur l'humanité de la folie, Paris, Seuil, 2014, p. 21.

10. F. Rigaux, Plaisir, interdits et pouvoir. Une analyse des discriminations fondées sur le sexe, l'orientation sexuelle et la race, Anvers, Kluwer, 20oo, p. VIII 